status, last-minute booster doses required, and the number of emails sent by the assessor in processing the records. The number of phone calls made and received were not recorded.

Results: To complete the skills matrix for a field hospital containing an emergency department and operating theater (an EMT type 2), 61 members were nominated. At the time of assessment, 32 (52\%) were fully immunized, requiring no further booster doses (vaccinations or serology tests). Three members were removed from the deployment as they were not fully immunized. Last-minute booster doses were required by 27 (44\%) members, with a total of 74 booster doses administered (range 0-5). 19 of the booster doses administered were immunizations required to work in any health facility in Australia. The most common vaccines requiring booster doses were rabies $(n=21)$ and typhoid $(n=15) .58$ emails were sent over a period of 5 days to 24 members to clarify vaccination status.

Discussion: This deployment highlighted a gap in members' perception of their immunization status, leading to delays in deployment readiness for the team. A new electronic system where vaccine status tracking occurs in real time should address this issue.

Prehosp Disaster Med 2019;34(Suppl. 1):s137-s138

doi:10.1017/S1049023X19003030

\section{The Impact of the New European Union General Data Protection Regulation (GDPR) on Data Collection at Mass Gatherings \\ Dr. Annelies Scholliers ${ }^{1,2}, \mathrm{Mr}$. Dimitri De Fre ${ }^{2,3}$, Mrs. Inge D'haese ${ }^{2}, M r$. Stefan Gogaert ${ }^{2}$ \\ 1. Department of Anaesthesiology and Perioperative Medicine, University Hospital Brussels, Jette, Belgium \\ 2. Mass Gathering Solutions, Wambeek, Belgium \\ 3. University Hospital Gasthuisberg, Leuven, Belgium}

Introduction: As of May 2018, a new European privacy law called the General Data Protection Regulation (GDPR) is in order. With this law, every organization operating in the European Union (EU), needs to adhere to a strict set of rules concerning collection and processing of personal data.

Aim: To explore the consequences of the GDPR for data collection at mass gatherings in the European Union.

Methods: Since the law was published on April 27, 2016, a thorough reading of the law was conducted by 4 persons with a background in mass gathering health. The GDPR consists of 99 articles organized into 11 chapters. There are also 173 recitals to further explain certain ambiguities. Key articles and recitals relating to healthcare and scientific research were identified. Possible pitfalls and opportunities for data collection and processing at mass gatherings were noted.

Discussion: Under article 4, key definitions are noted. There is a clear definition of "data concerning health". According to the GDPR, health data is a special category of personal data which should not be processed according to article 9(1). However, there is an exception for scientific research (article $9(2)(j))$. There are a few safeguards in place, as laid out in article 89. One interesting point is that according to article $89(2)$, certain derogations can take place if the law interferes with scientific research. The GDPR has major consequences for data collection and processing in the EU. However, with the use of certain safeguards (e.g., pseudonymization) there are still ample opportunities for scientific research. It is important to review one's method of data collection to make sure it complies with the GDPR.

Prehosp Disaster Med 2019;34(Suppl. 1):s138

doi:10.1017/S1049023X19003042

Impact Scale for the Continuity of Care in Contingency Management Situations - Operationalization of the Crisis Standards of Care

Mr. Roel Geene ${ }^{1}$, Dr. Pieter van der Torn ${ }^{2}$, Dr. Dennis den

Hartog ${ }^{3}$

1. Trauma Centre Southwest, Erasmus MC, University Medical Center Rotterdam, Rotterdam, The Netherlands

2. Itineris Consultancy, Rotterdam, The Netherlands

3. Trauma Research Unit, Department of Surgery, Erasmus MC, University Medical Center Rotterdam, Rotterdam, The Netherlands

Introduction: A common language is needed to compare the impacts of incidents, crises, and disasters among health care coalition members, such as emergency medical services, general practitioners, and hospitals. A generic impact scale was developed, based on the Crisis Standards of Care, and was put to the test during the 2017 and 2018 winter/flu-seasons.

Aim: To define an impact scale for the quantitative assessment of the hospital response to incidents, crises, and disasters.

Methods: An impact scale has to be generally applicable to be useful in the context of a health care coalition. It should be applicable to all hazards and all parties in proactive and reactive, real-time settings. In addition, the scale should be easy to understand and score and should be independent of the various information systems in use. The Crisis Standards of Care were chosen as basis and were operationalized in a seven-point Likert-scale for expert-based scoring: "No impact," "Buffer capacity needed," "Buffer capacity sufficient," "Unusual adaptations to care needed," "Unusual adaptations sufficient," "Disturbance of continuity of care inevitable without external assistance," and "Disturbance of continuity of care inevitable." Results: During the 2017 and 2018 winter/flu-seasons, crisis managers of ten hospitals scored the scale almost daily for three months. This served as a regional monitor and created the possibility to distribute patients and resources more evenly over the hospitals and with the care sector.

Discussion: The impact scale improved communication and mutual understanding between hospitals and with other health care organizations, and is expected to have helped in maintaining the continuity of care during the 2017 and 2018 winter/ flu-seasons. More research is needed on the reliability of the response. Nevertheless, the scale has since become an integral part of the regional contingency planning.

Prehosp Disaster Med 2019;34(Suppl. 1):s138

doi:10.1017/S1049023X19003054

Implementing Guidelines for Ambulance Services Dr. Rubije Hodza-Beganovic, Mr. Henrik C. Carlsson, Mr. Henrik Lidberg, Dr. Peter Berggren

KMC/IMP, Linköping, Sweden 